tienten mit chronischen Schmerzen. Die meisten waren von mäßiger bis mittelmäßiger Qualität, meist wurden Pregabalin, Gabapentin und Topiramat geprüft. Die Resultate waren eher negativ bei nicht radikulären und widersprüchlich bei radikulären Schmerzen. Letztlich sei die Evidenz jedoch ungenügend für eine Beurteilung, konstatieren die Ärzte.

Systemische Kortikoide: Die zehn ausgewerteten Studien führten zu überwiegend negativen Resultaten. Weder Injektionen noch Tabletten schnitten bei akuten Schmerzen wesentlich besser ab als Placebo, auch funktionelle Verbesserungen waren eher gering. „Systemische Kortikoide scheinen nicht wirksam zu sein“, schlussfolgern die Forscher um Chou.
Fazit: Insgesamt fällt das Ergebnis der Analyse also mager aus. Die meisten Substanzklassen hätten zwar eine gewisse Wirksamkeit bei akuten oder chronischen Rückenschmerzen, aber meist sei diese kurzfristig und eher gering. Paracetamol und Trizyklika könne man aufgrund neuer Daten getrost von der Liste der wirksamen Optionen streichen, Duloxetin neu hinzufügen, wenngleich auch hier die Schmerzlinderung eher gering ausfalle. Nur für NSAR, Opioide und Duloxetin deuteten die Studien auf einen geringen, gelegentlich moderaten Effekt bei chronischen Kreuzschmerzen.

Entsprechend zurückhaltend gibt sich auch die aktualisierte ACP-Leitlinie. „Ärzte sollten Patienten mit akuten oder subakuten Schmerzen im unteren Rückenbereich darauf hinweisen, dass die Schmerzen in der Regel wieder von selbst verschwinden“, erläutert der ACPVorsitzende Dr. Nitin Damle in einer Mitteilung der Gesellschaft. „Sie sollten auch unnötige Tests sowie teure oder potenziell schädliche Medikamente vermeiden." (www.acponline.org/acp-newsroom)

Bei chronischen Kreuzschmerzen raten die US-Leitlinien primär zu mehr körperlicher Bewegung, Gymnastik, multidisziplinären und nicht medikamentösen Verfahren mit Akupunktur, Yoga oder Stressreduktion. Genügt das nicht, sollten Ärzte eine Behandlung mit NSAR erwägen. Opioide seien möglichst zu meiden.

Thomas Müller

Chou R et al. Systemic Pharmacologic Therapies for Low Back Pain: A Systematic Review for an American College of Physicians Clinical Practice Guideline. Ann Intern Med 2017; 166(7):480-92

\title{
Postoperative Schmerzen nach Herz-OP: Nach einem Jahr ist das Schlimmste überstanden
}

\author{
Rund $10 \%$ der Patienten nach einer Herzoperation klagen über chronische, \\ mindestens zwei bis drei Monate anhaltende Schmerzen, die die Lebensqua- \\ lität beeinträchtigen. Wie sieht die weitere Entwicklung aus? Eine prospektive \\ 5-Jahres-Studie macht den Patienten Mut.
}

$\mathrm{D}$ ie Inzidenz chronischer Schmerzen nach Herzoperationen wird mit 10$56 \%$ angegeben. Die hohe Varianz lässt sich mit Unterschieden bei der Definition von chronischen postoperativen Schmerzen (chronic postsurgical pain, CPSP), der Auswahl der Studienteilnehmer und einem prospektiven vs. retrospektiven Design erklären. Unsicherheit besteht bei dieser Fragestellung auch bezüglich des langfristigen CPSP-Verlaufs. Eine große prospektive 5-Jahres-Studie aus Trondheim schafft Klarheit.

Einbezogen in die monozentrische Kohortenstudie waren $534 \mathrm{zu}$ einer Herzoperation anstehende Patienten, davon $23 \%$ über 75 Jahre alt. Unmittelbar nach der Operation beantworteten die Patienten erstmalig Fragen zur Lebensqualität per Short-Form Health Survey (SF-36) Version 1.2 sowie zum Schmerzstatus per Brief Pain Inventory (BPI). Nach einem und nach fünf Jahren erhielten sie die
Fragebögen erneut zugeschickt. Ein CPSP wurde dann angenommen, wenn die Schmerzen erst nach der Operation erstmalig aufgetreten waren und mindestens über drei Monate anhielten.

Von 458 nach fünf Jahren noch lebenden Patienten absolvierten 373 (82\%) alle drei Fragedurchläufe. 89,8\% berichteten weder nach einem noch nach fünf Jahren über CPSP. Bei $63 \%$ der 38 Patienten mit CPSP nach einem Jahr waren die Schmerzen in der erneuten Befragung vier Jahre später verschwunden, sodass sich eine 5-Jahres-Prävalenz von 3,8\% ergab. $\mathrm{Pa}$ tienten mit CPSP ein beziehungsweise fünf Jahre nach der Herzoperation hatten eine schlechtere Lebensqualität und berichteten vermehrt über Schmerzen bereits vor der Operation.

Fazit: Die in einer prospektiven Kohortenstudie ermittelte 5-Jahres-Prävalenz chronischer postoperativer Schmerzen

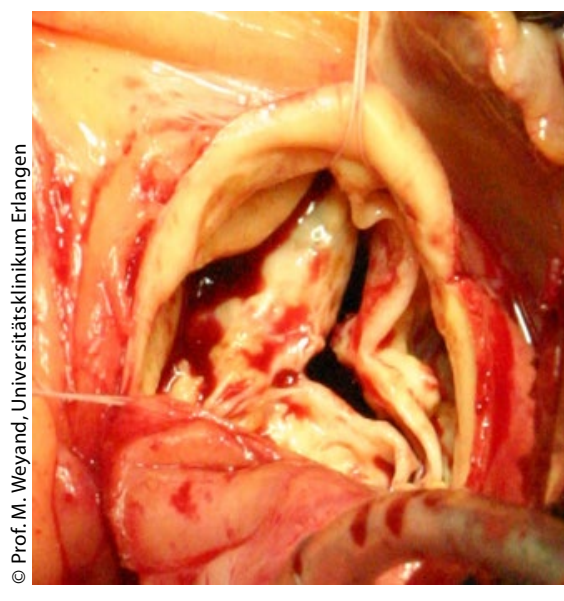

Eingriff bei Stenose der Aortenklappe

nach Herzchirurgie beträgt 3,8\% und liegt damit deutlich niedriger als bisherige Schätzungen, die allerdings überwiegend auf retrospektiven Befragungen beruhten. Bei $63 \%$ der Patienten, die ein Jahr nach der Herzoperation noch CPSP angaben, war die Symptomatik nach fünf Jahren verschwunden. Risikofaktor für ein CPSP-Syndrom ist unter anderem eine positive präoperative Schmerzanamnese.

Dr. Barbara Kreutzkamp

Gjeilo $\mathrm{KH}$ et al. Chronic postsurgical pain in patients 5 years after cardiac surgery: A prospective cohort study. Eur J Pain 2017;21:425-33 